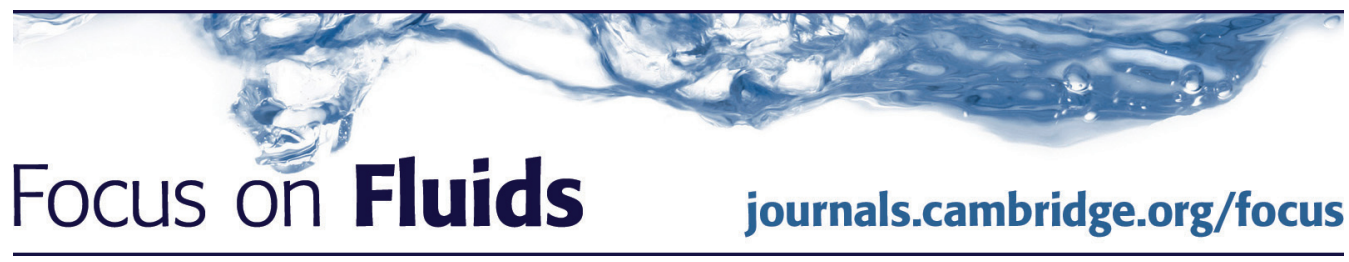

\title{
Micro-plumes for \\ nano-velocimetry
}

\author{
Todd M. Squires $\dagger$ \\ Department of Chemical Engineering, University of California, \\ Santa Barbara, CA 93106-5080, USA
}

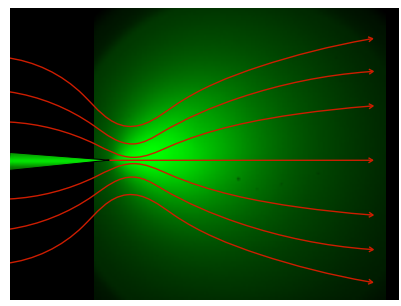

Fluid flows through nano-scale channels depend sensitively on the physical and chemical properties of the walls that surround them. The sub-micron dimensions of such channels, however, are impossible to resolve optically, which rules out most methods for flow visualization. Classic calculations by Squire (Q. J. Mech. Appl. Maths, vol. IV, 1951, pp. 321-329) and Landau \& Lifshitz (Fluid Mechanics, vol. 6, 1959, Pergamon) showed that the laminar flow driven outside a capillary, by fluid emerging from the end of the capillary, is identical to the flow driven by a point force proportional to the average velocity in the capillary. Secchi et al. (J. Fluid Mech. 826, R3) analyze the dispersion of a solute that is injected along with the fluid, whose concentration decays slowly with distance but with a strong angular dependence that encodes the intra-capillary velocity. Fluorescence micrographs of the concentration profile emerging from the nanocapillary can be related directly to the average fluid velocity within the nanocapillary. Beyond their remarkable capacity for nano-velocimetry, Landau-Squire plumes will likely appear throughout micro- and nano-fluidic systems.

Key words: jets, low-Reynolds-number flows, micro-/nano-fluid dynamics

\section{Introduction}

Several decades of research involving 'microfluidics' - microfabricated fluidic devices with 10-100 $\mu \mathrm{m}$ dimensions - have enabled advances in both technology and science. For example, one advanced technique for DNA analysis uses microfluidic devices to generate and manipulate sample and reagent droplets. In parallel, exquisite control over microfluidic geometries and conditions have enabled fundamental scientific studies, large-scale screens of materials and phenomena, and synthetic mimics of natural systems that would be otherwise difficult or impossible to probe (Squires \& Quake 2005).

Later came nanofluidic systems (Schoch, Han \& Renaud 2008; Bocquet \& Tabeling 2014), whose even smaller length scales bring different physical phenomena to prominence. As device dimensions shrink, increasing fractions of the flowing fluids

$\dagger$ Email address for correspondence: squires@engineering.ucsb.edu 

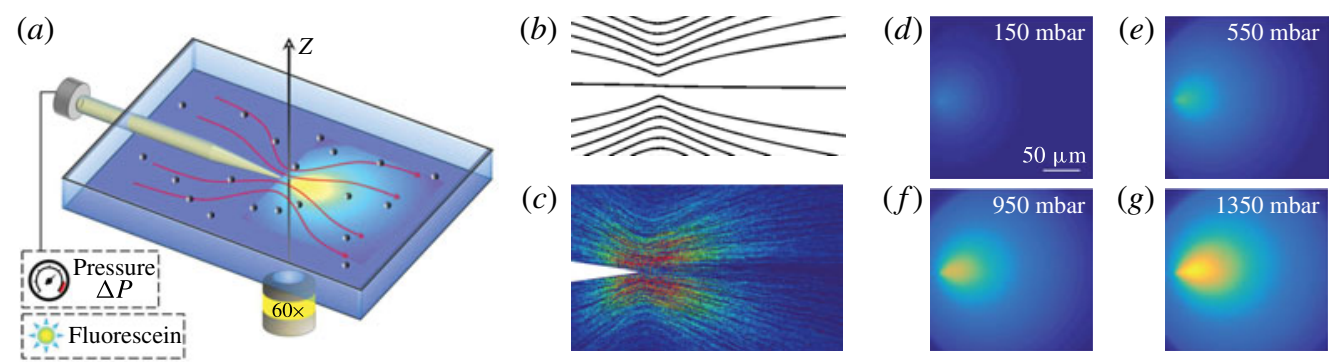

FIGURE 1. (a) A nanocapillary injects fluorescein into a fluid reservoir. (b) Velocity field outside a nanocapillary, driven by emerging fluid, as computed by Squire (1951) and Landau \& Lifshitz (1959). (c) Particle-tracking velocimetry agrees well with Landau-Squire flows. $(d-g)$ Fluorescence measurements of fluorescein dispersion due to Landau-Squire flow. Landau-Squire plumes become more anisotropic and jet-like $(g)$ with increasing injection velocity.

are affected by physico-chemical interactions with the surrounding walls. Nanofluidic channels might be smaller than the Navier slip length, or the electric double-layer thickness, changing flow properties qualitatively (Schoch et al. 2008). In fact, the nearness of walls in nanofluidic systems has enabled a nanofluidic complement to microfluidic DNA sequencing: individual DNA molecules can now be 'read' as they pass through nano-pores (Bocquet \& Tabeling 2014).

A variety of difficulties arise as fluidic pipes shrink. One, in particular, may seem excruciatingly mundane: determining fluid velocities in nanofluidic channels. A suite of velocimetry techniques have been developed for microchannels - e.g. tracking tracer particles, or micro-particle image velocimetry algorithms designed to accomodate the stochastic nature of Brownian tracer particles (Werely \& Meinhart 2010). Nanofluidic channels, however, are typically much too thin to be resolved optically, significantly impeding particle tracking. Moreover, smaller channels require smaller particles, strengthening their Brownian motion and smearing their positions during optical aquisition. Wall interactions may bias the distribution of tracer particles within the channel - and therefore the inferred flows.

Last year, Secchi et al. (2016) provided an elegant solution to this practical problem. To measure fluid velocities through carbon nanotubes (CNT) and boron nitride nanotubes (BNNT), they exploited a novel feature of the laminar flow that emerges from a small capillary into an infinite fluid (figure 1a), first computed by Squire (1951) and Landau \& Lifshitz (1959). In particular, the Landau-Squire flow outside a nanotube of radius $R$ is identical to that due to a point force of magnitude $F_{L S} \sim \bar{V} R$, where $\bar{V}$ is the average velocity inside the nanotube (figure $1 b$ ). By measuring the flow outside the nanotube - far easier, given the hundreds of micron length scales involved - Secchi et al. (2016) determined the force $F_{L S}$ responsible for the observed Landau-Squire flows, from which the intra-capillary velocity $\bar{V}$ followed directly. Underscoring the importance of wall effects in nanofluidic systems, they found the flow through BNNTs to obey the standard no-slip condition, but flow through CNTs to exhibit strong radius-dependent slip at the solid-liquid surface.

\section{Overview}

Building upon this foundation, Secchi et al. (2017) now present what is nominally an even simpler approach to capillary velocimetry. In so doing, they highlight an 
elegant solution to a paradigmatic micro- and nano-fluidic scenario - the dispersion of solutes injected from a capillary. The injected solute convects along with the LandauSquire flow, and diffuses within it, to form a plume whose shape encodes the injection velocity, as exploited by Secchi et al. (2017) for intra-capillary velocimetry.

Secchi et al. (2017) expand upon and further develop Squire's (1951) solution to the convection-diffusion problem, by providing an intuitive expression that highlights the impact of an effective Peclet number,

$$
P e=\frac{\bar{V} R}{D_{s}},
$$

on plume shape, where $D_{s}$ is the solute diffusivity. $P e$ represents the relative importance of convective versus diffusive transport. Low-Pe Landau-Squire plumes are relatively symmetric (figure $1 d$ ), whereas high- $P e$ plumes are jet-like and concentrated along the downstream axis (figure $1 g$ ). Moreover, the high- $P e$ concentration field depends exponentially on $P e$, implying that the plume's shape (which is relatively easy to measure) depends sensitively on its ejection velocity $\bar{V}$ (which is much more difficult to measure).

Exquisite experiments confirm their strategy. Secchi et al. injected fluorescent solutions through nano-scale capillaries at various imposed pressures (figure 1a), and measured the concentration field in the plume using fluorescence microscopy (figure $1 d-g$ ). Concentration fields measured at different imposed pressures agree quantitatively with those computed analytically, at the Peclet number appropriate for each plume shape. In this way, the appropriate $P e$ can be determined for each plume, which therefore reveals the fluid velocity $\bar{V}$ within the nanocapillary. Further validation is provided by direct, particle-tracking velocimetry of the (Landau-Squire) flow outside the capillary (figure 1c).

\section{Future}

This work suggests a variety of interesting future directions, beyond the new-found ease of nanocapillary velocimetry. Before moving to those, however, it is worth pondering what nanocapillary velocimetry itself enables. Indeed, the particle-tracking strategy had already revealed a truly remarkable difference in the liquid-solid slip condition between nanotubes of different molecular compositions (Secchi et al. 2016). Nanotubes and nano-capillaries may well provide a wealth of information about how the physical and chemical properties of capillary surfaces impact the fluids that flow over them. For example, one may probe the effect of surface charge densities, surface chemical modifications, grafted or adsorbed polymers, proteins, surfactants, or nano-bubbles on fluid flows. Similarly, the properties of the solution itself - e.g. ionic strength, ion valence, or $\mathrm{pH}$ - may modify those surface properties, and therefore change fluid fluxes in a measurable way. Complex fluids that contain polymers, proteins, micelles, or particles may self-organize, shear and flow very differently under the strong confinement of nanocapillaries or nanotubes. Likewise, other manners of driving flows - e.g. electro-osmosis, diffusio-osmosis, temperature or solvent gradients, or osmotic pressure differences - may be studied in this fashion. It is hard to imagine an easier method to probe such flows within nanometric channels.

Beyond velocimetry, Landau-Squire plumes may influence colloids suspended in the surrounding fluid. In particular, colloids migrate diffusiophoretically in response to gradients in solute concentration $c$ (Prieve et al. 1984; Derjaguin, Dukhin \& 
Korotkova 1993), and microfluidic systems have proven quite useful for the control and study of diffusiophoresis (Abecassis et al. 2008; Paustian et al. 2015). Gradients arise for many reasons (Velegol et al. 2016), implying that diffusiophoresis occurs in a wide range of micro- and nano-fluidic processes (e.g. Florea et al. 2014; Banerjee et al. 2016; Shin et al. 2016). Because diffusiophoresis often scales with the logarithmic derivative of concentration, the exponential angular dependence of high-Pe Landau Squire plumes suggests diffusiophoresis will remain strong but localized around the jet.

Finally, Secchi et al. (2017) have rightly highlighted a paradigmatic flow for the micro- and nano-fluidic world, which now seems like one we should already have held in mind. The linearity of low- $R e$ flows allows intuition from a single plume to guide the conceptual design of systems with multiple capillaries, each with its own flow rate. Although solute dispersion from multiple capillaries does not superpose in the same way, the Landau-Squire plume provides key intuitive guidance.

\section{References}

Abecassis, B., Cottin-Bizonne, C., Ybert, C., Ajdari, A. \& Bocquet, L. 2008 Boosting migration of large particles by solute contrasts. Nat. Mater. 7 (10), 785-789.

Banerjee, A., Williams, I., Azevedo, R. N., Helgeson, M. E. \& Squires, T. M. 2016 Soluto-inertial phenomena: designing long-range, long-lasting, surface-specific interactions in suspensions. Proc. Natl Acad. Sci. USA 113 (31), 8612-8617.

Bocquet, L. \& TABeling, P. 2014 Physics and technological aspects of nanofluidics. Lab on a Chip 14, 3143-3158.

Derjaguin, B. V., Dukhin, S. S. \& Korotkova, A. A. 1993 Diffusiophoresis in electrolyte solutions and its role in the mechanism of the formation of films from caoutchouc latexes by the ionic deposition method. Prog. Surf. Sci. 43 (1-4), 153-158.

Florea, D., Musa, S., Huyghe, J. M. R. \& Wyss, H. M. 2014 Long-range repulsion of colloids driven by ion exchange and diffusiophoresis. Proc. Camb. Phil. Soc. 111 (18), 6554-6559.

Landau, L. D. \& Lifshitz, E. M. 1959 Fluid Mechanics, vol. 6. Pergamon.

Paustian, J. S., Angulo, C. D., Nery-Azevedo, R., Shi, N., Abdel-Fattah, A. I. \& Squires, T. M. 2015 Direct measurements of colloidal solvophoresis under imposed solvent and solute gradients. Langmuir 31, 4402-4410.

Prieve, D. C., Anderson, J. L., Ebel, J. P. \& Lowell, M. E. 1984 Motion of a particle generated by chemical gradients. Part 2. Electrolytes. J. Fluid Mech. 148, 247-269.

Schoch, R., Han, J. \& Renaud, P. 2008 Transport phenomena in nanofluidics. Rev. Mod. Phys. 80 (3), 839-883.

Secchi, E., Marbach, S., Niguès, A., Siria, A. \& Bocquet, L. 2017 The Landau-Squire plume. J. Fluid Mech. 826, R3.

Secchi, E., Marbach, S., Niguès, A., Stein, D., Siria, A. \& Bocquet, L. 2016 Massive radius-dependent flow slippage in carbon nanotubes. Nat. Biotechnol. 537 (7619), 210-213.

Shin, S., Um, E., Sabass, B., Ault, J. T., Rahimi, M., Warren, P. B. \& Stone, H. A. 2016 Size-dependent control of colloid transport via solute gradients in dead-end channels. Proc. Natl Acad. Sci. USA 113 (2), 257-261.

SQuire, H. B. 1951 The round laminar jet. Q. J. Mech. Appl. Maths IV, 321-329.

SQuires, T. M. \& QUAKE, S. R. 2005 Microfluidics: fluid physics at the nanoliter scale. Rev. Mod. Phys. 77 (3), 977-1026.

Velegol, D., Garg, A., Guha, R., Kar, A. \& Kumar, M. 2016 Origins of concentration gradients for diffusiophoresis. Soft Matt. 12 (21), 4686-4703.

Werely, S. T. \& Meinhart, C. D. 2010 Recent advances in micro-particle image velocimetry. Annu. Rev. Fluid Mech. 42, 557-576. 